Noname manuscript No.

(will be inserted by the editor)

\title{
Conflicts among $N$ armed groups: Scenarios from a new descriptive model
}

\author{
Sergio Rinaldi • Fabio Della Rossa
}

Received: date / Accepted: date

\begin{abstract}
In this paper we propose and analyze a new descriptive model of armed conflicts among $N$ groups. The model is composed of $N^{2}$ ordinary differential equations, with $3\left(N^{2}+N\right)$ constant parameters that describe military characteristics and recruitment policies, ranging from pure defensivism to pure fanaticism. The results are only preliminary, but point out interesting (though not very surprising) properties: periodic coexistence is possible, and multiple attractors can exist; governmental groups cannot go extinct if they are highly defensivist, and rebels cannot be eradicated if they are highly fanatic. Shocks due to interventions of short duration of an external army can stabilize/destabilize the system and/or eradicate some group, and the same holds true for small structural changes. Other more subtle questions concerning, for example, the existence of chaotic regimes and the systematic evaluation of the role of strategic factors like power, intelligence, and fanaticism, remain open and require further research.
\end{abstract}

Keywords mathematical modeling $\cdot$ social systems $\cdot$ conflicts $\cdot$ terrorism $\cdot$ bifurcations $\cdot$ chaos

\section{Introduction}

Conflicts between two armed groups have been studied with paradigmatic models (i.e., simple allegories of the real world) for more than one century (see [1] for a survey). The models are of three different types:

- descriptive (ordinary differential equations),

- individual based (interactions among groups of individual units),

- rational agents (mathematical programming, optimal control, and game theory).

Models of the first class, composed in general of two differential equations (one for each group, see [4] for a recent survey), combine and extend the pioneering studies of Lanchester [2] and Richardson [5,6]. They show that the fight does not necessarily end with the annihilation of one group. Indeed, in these models stationary coexistence of the groups (so-called stationary stalemate) has been shown to be possible. As proved by experience and discussed in recent and outstanding survey papers (see [1] and [4]), descriptive models are of great conceptual value even if they can rarely be applied to specific real cases.

Here we propose a new and more sophisticated descriptive model in which individuals are either searching for enemies or handling prisoners, and groups can follow different recruitment policies ranging from pure defensivism to pure fanaticism. With this model we show that also periodic coexistence is

Sergio Rinaldi

Dipartimento di Elettronica, Informazione e Bioingegneria, Politecnico di Milano, Milan, Italy

International Institute for Applied Systems Analysis, Laxenburg, Austria

Tel.: +390223993563

E-mail: sergio.rinaldi@polimi.it

Fabio Della Rossa

Dipartimento di Elettronica, Informazione e Bioingegneria, Politecnico di Milano, Milan, Italy

Tel.: +390223993579

E-mail: fabio.dellarossa@polimi.it 
possible, thus supporting the idea that conflicts can be characterized by recurrent ups and downs in the dominance of the groups.

Our model can deal with the general case of $N$ groups fighting one against the other, a problem of increasing concern. The model is composed of $N^{2}$ ordinary differential equations, containing $3\left(N^{2}+N\right)$ parameters, used to mimic the recruitment policies as well as the offensive and intelligence efficiencies of the groups. Since equations and parameters increase in number as $N^{2}$, the study of the model requires a remarkable effort even if it is limited, as done in this paper, to the case of 3 fighting groups.

The work performed so far along this line is still in progress. For this reason, we present only preliminary results pointing out, however, a number of interesting behaviors. In particular we explain why external interventions of short duration (shocks) can modify the fate of the conflict, and why small but permanent (structural) changes can trigger the annihilation of a group or transform a stationary stalemate into a cyclic one.

\section{Behavioural characteristics}

We consider a finite number of groups $i=1, \ldots, N$ fighting one against the other. The size of each group at time $t$ is denoted with $x_{i}(t)$, and the individuals of the group are assumed to be, at any time $t$, either searching for enemies or handling prisoners. Thus

$$
x_{i}(t)=s_{i}(t)+h_{i}(t)
$$

where $s_{i}(t)$ are all individuals of group $i$ searching for enemies (of any group), and $h_{i}(t)$ are handling individuals of group $i$. Handling includes all activities that follow the capture of an enemy, namely the elimination of the victims, the transportation, supervision and feeding of the prisoners, their exchange for ransom, and so on. These service activities require some time called handling time. An individual who has accomplished all handling tasks becomes searching again (transition $h \rightarrow s$ ), while a searching individual becomes handling immediately after capturing an enemy (transition $s \rightarrow h$ ).

Since handling activities can be different for different groups of prisoners, we denote with $h_{i j}$ the individuals of group $i$ handling prisoners of group $j$ and with $\tau_{i j}$ their handling times. Obviously

$$
h_{i}(t)=\sum_{j} h_{i j}(t)
$$

\section{Capture of enemies}

When searching individuals of group $i$ discover an enemy, they attack and try to capture the enemy, but their success depends not only on the group to which the enemy belongs but also on the fact that the discovered enemy is searching or handling. The rates at which searching individuals of group $i$ encounter and capture searching and handling individuals of group $j$ are indicated with $\sigma_{i j}^{s}$ and $\sigma_{i j}^{h}$ respectively. If the searching process is purely random, the standard assumption is that $\sigma_{i j}^{s}$ and $\sigma_{i j}^{h}$ are independent upon the size of the searching group. But more sophisticated assumptions could also be considered. For example, if searching individuals of group $i$ are cooperative (e.g., if they communicate and call each other when they discover groups of enemies) it would be reasonable to assume that $\sigma_{i j}^{s}$ and $\sigma_{i j}^{h}$ increase with the size of the searching group. But also the case of $\sigma_{i j}^{s}$ and $\sigma_{i j}^{h}$ decreasing with the size of the searching group could be interesting because it interprets the situation in which soldiers of the same group interfere negatively. In general, the success rates $\sigma_{i j}^{s}$ and $\sigma_{i j}^{h}$ are different and $\sigma_{i j}^{s}>\sigma_{i j}^{h}$ because handling is often performed in relatively hidden or protected areas. But in special circumstances, in particular when the intelligence of group $i$ is high, safe refuges do not exist for group $j$, and $\sigma_{i j}^{s}=\sigma_{i j}^{h}$. Notice that, in order to reduce the number of parameters, individuals of group $i$ are assumed to have the same success with all handling individuals of group $j$. Moreover, all captured individuals are eliminated from the game, i.e., prisoners can not be freed or, if they are, they do not return immediately to their groups.

Let us now indicate with $V_{i j}$ the flow of victims of group $j$ captured by (searching) individuals of group $i$. From now on, when there are two indexes (say $i$ and $j$ ), the first indicates the group of the killer 
and the second the group of the victim. Since individuals are either searching or handling when they are captured, we must consider two flows, namely $V_{i j}^{s}$ and $V_{i j}^{h}$. Obviously

$$
V_{i j}=V_{i j}^{s}+V_{i j}^{h} .
$$

We can also introduce the flows

$$
V_{i+}^{s}=\sum_{j} V_{i j}^{s}, \quad V_{i+}^{h}=\sum_{j} V_{i j}^{h},
$$

of all searching individuals captured by group $i$ and of all handling individuals captured by group $i$ and indicate with $V_{i+}$ the flow of all individuals captured by group $i$, i.e.,

$$
V_{i+}=V_{i+}^{s}+V_{i+}^{h} .
$$

Similarly we can consider the flows

$$
V_{+j}^{s}=\sum_{i} V_{i j}^{s}, \quad V_{+j}^{h}=\sum_{i} V_{i j}^{h}
$$

of searching or handling individuals of group $j$ captured by some enemy and indicate with

$$
V_{+j}=V_{+j}^{s}+V_{+j}^{h}
$$

the flow of all victims of group $j$.

All flows are known once the flows $V_{i j}^{s}$ and $V_{i j}^{h}$ are specified as functions of the sizes of the searching and handling groups. In the following we consider the case of random encounters where

$$
V_{i j}^{s}=\sigma_{i j}^{s} s_{i} s_{j}, \quad V_{i j}^{h}=\sigma_{i j}^{h} s_{i} h_{j} .
$$

\section{Recruitment policies}

We assume that each group is sustained by suitable recruitments (e.g., soldiers during their military service, voluntiers, hired professionals, ...) and we distinguish between two different flows of recruitment.

The first, indicated with $G_{i}$ and called guarandeed recruitment, is a flow supported by the state or its allies independently on the current injuries suffered by the group or inflicted to its enemies. This flow is simply a function of the size of the group, i.e., $G_{i}=G_{i}\left(x_{i}\right)$ and the selected functional form is

$$
G_{i}\left(x_{i}\right)=r_{i} x_{i}-c_{i} x_{i}^{2}
$$

where $r_{i} x_{i}$ is the difference between basic recruitment and basic retirement when the size of the group is small, while $c_{i} x_{i}^{2}$ is the surplus of retirement present when difficulties in the progress of careers or limitations in salaries and benefits emerge when the group becomes large. In general, $r_{i}>0$ and $c_{i}>0$ so that the maximum guaranteed recruitment is $r_{i}^{2} / 4 c_{i}$. But $r_{i}$ can also be negative: this occurs when basic retirement of small groups overcomes basic recruitment, i.e., when the group goes gradually extinct in the absence of enemies. This might interpret the dynamics of groups of fanatic rebels.

The second form of recruitment, indicated with $R_{i}$ and called reaction recruitment, is a function of the current injuries suffered by the group $\left(V_{+i}\right)$ and inflicted to its enemies $\left(V_{i+}\right)$. In the following, for simplicity, we assume that the reaction recruitment $R_{i}$ depends on a weighted sum $z_{i}$ of the suffered and inflicted injuries, i.e., $R_{i}=R_{i}\left(z_{i}\right)$, where

$$
z_{i}=d_{i} V_{+i}+f_{i} V_{i+} .
$$

In this sum the two weights $d_{i}$ and $f_{i}$ are assumed to be constant and are called defensivism and fanaticism, respectively. Groups representing the army or anti-terrorism services of standard countries have $f_{i}=0$ (or very small), while $f_{i}$ can be large in groups of fanatic rebels. If group $i$ has different reactions with respect to different groups we should use the more general weighted sum

$$
z_{i}=\sum_{j} d_{i j} V_{j i}+\sum_{j} f_{i j} V_{i j}
$$


where defensivism and fanaticism depend on two indexes. This is certainly possible, but is not done here in order to keep the complexity of the model under control. The function $R_{i}\left(z_{i}\right)$ must be zero for $z_{i}=0$, because in absence of injuries the recruitment must be the guaranteed recruitment. Moreover, it is reasonable to assume that the function $R_{i}\left(z_{i}\right)$ is increasing with $z_{i}$, since the reaction to injuries obviously increases with injuries. Finally, the function $R_{i}\left(z_{i}\right)$ must saturate for $z_{i} \rightarrow \infty$, because unbounded recruitments can not be realized in view of physical and economic constraints. Figure 1 shows a reaction recruitment that satisfies the above three properties. The function $R_{i}\left(z_{i}\right)$ used in the model is

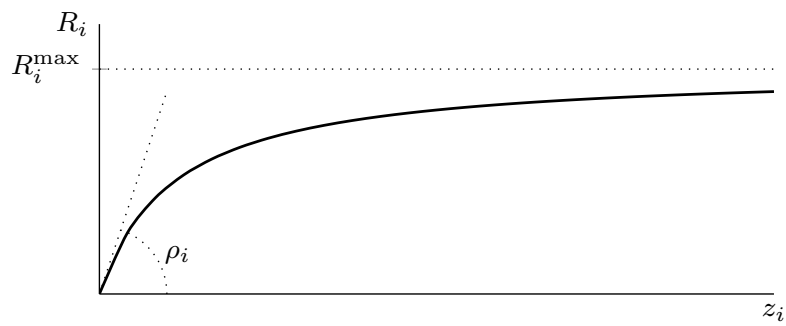

Fig. 1 Reaction recruitment as a function of the weighted sum $z_{i}$ of the injuries. The graph is obtained from equation (11) with $R_{i}^{\max }=1, \rho_{i}=3$.

$$
R_{i}\left(z_{i}\right)=\frac{\rho_{i} R_{i}^{\max } z_{i}}{\rho_{i} z_{i}+R_{i}^{\max }}
$$

where the meaning of the two parameters $\rho_{i}$ and $R_{i}^{\max }$ is pointed out in Figure 1.

In order to fully specify the model, we must also say how the recruitment $\left(G_{i}+R_{i}\right)$ is distributed among searching and handling groups. If we denote with $\lambda_{i}^{s}$ the portion of recruitment allocated to the searching group and with $\lambda_{i j}^{h}$ the portion allocated to the group handling individuals $j$ (obviously $\lambda_{i}^{s}+\sum_{j} \lambda_{i j}^{h}=1$ ), in principle various options are possible. For example, we could assume that the recruitment is entirely allocated to the searching group, i.e., $\lambda_{i}^{s}=1, \lambda_{i j}^{h}=0$, or that it is equally subdivided among all groups, i.e., $\lambda_{i}^{s}=\lambda_{i j}^{h}=1 / N$, ot that it is subdivided proportionally to the sizes of the groups, i.e., $\lambda_{i}^{s}=s_{i} / x_{i}, \lambda_{i j}^{h}=h_{i j} / x_{i}$. Here we follow the third option, because it is the only one that can be shown to guarantee the positivity of the model (i.e., the property that starting from positive initial conditions the state of the system remains positive forever).

\section{A general model}

The model is fully specified by writing the balance equations for each searching and handling group. Figure 2 shows the flows of individuals joining and leaving each group. For each searching group we must

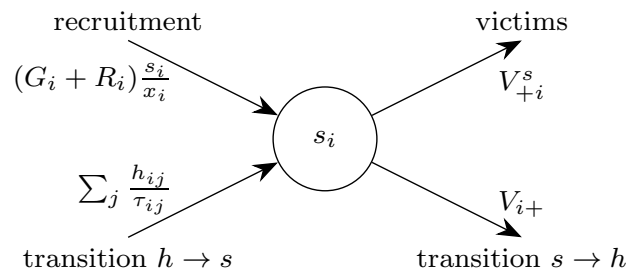

(a)

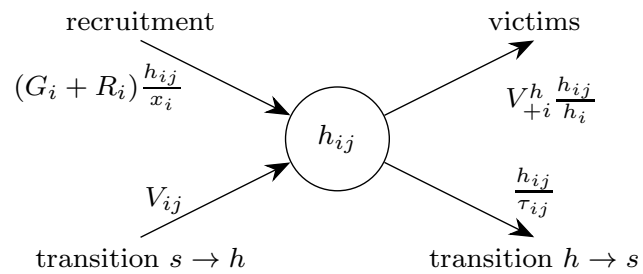

(b)

Fig. 2 The flows of individuals joining and leaving each searching group (a) and each handling group (b).

write the equation (see Figure 2a)

$$
\dot{s}_{i}=\left(G_{i}\left(x_{i}\right)+R_{i}\left(z_{i}\right)\right) \frac{s_{i}}{x_{i}}+\sum_{j} \frac{h_{i j}}{\tau_{i j}}-V_{+i}^{s}-V_{i+}
$$


while for each handling group we have (see Figure $2 \mathrm{~b}$ )

$$
\dot{h}_{i j}=\left(G_{i}\left(x_{i}\right)+R_{i}\left(z_{i}\right)\right) \frac{h_{i j}}{x_{i}}+V_{i j}-V_{+i}^{h} \frac{h_{i j}}{h_{i}}-\frac{h_{i j}}{\tau_{i j}} .
$$

In these equations the flow $h_{i j} / \tau_{i j}$ is the flow of individuals of group $i$ terminating their handling tasks with individuals of group $j$, under the assumption of random termination of the tasks.

A set of $N$ ODEs for the group sizes can be easily derived from equations (12). Indeed, from equations $(1,2)$ we have

$$
\dot{x}_{i}(t)=\dot{s}_{i}(t)+\sum_{j} \dot{h}_{i j}(t)
$$

which, taking (12) into account, becomes

$$
\dot{x}_{i}=G_{i}\left(x_{i}\right)+R_{i}\left(z_{i}\right)-V_{+i} .
$$

Obviously this equation could be written a priori, because it simply says that the derivative of the group size is the unbalance between total flow of recruitment and flow of victims.

Thus, equation (12a) can be substituted by equation (13). In the following we refer to the set of equations $(1-11,12 \mathrm{~b}, 13)$ because it has the advantage of pointing out explicitly the sizes $x_{i}$ of the groups, which are undoubtedly variables of great concern.

Model $(1-11,12 \mathrm{~b}, 13)$ can hardly be studied analytically because, even in the simplest case $(N=2)$, it is composed of four nonlinear differential equations. However, three interesting general results can be proved. The first says that governmental groups (characterized by $r_{i}>0$ ) cannot go extinct if they are highly defensivist. Indeed, when the size $x_{i}$ is small, the flow of victims $V_{+i}$ and $V_{i+}$ are also small so that, in view of equations (9-11), we can approximate equation (13) as

$$
\dot{x}_{i} \simeq r_{i} x_{i}+\left(\rho_{i} d_{i}-1\right) V_{+i}+\rho_{i} f_{i} V_{i+} .
$$

Hence, if group $i$ is highly defensivist (in the sense that $\rho_{i} d_{i}>1$ ) $\dot{x}_{i}$ is positive for small $x_{i}$, and this prevents the extinction of the group. The second result concerns the groups of rebels (characterized by $\left.r_{i}<0\right)$ and says that none of them can achieve absolute victory since, in the absence of enemies $\left(x_{j}=0\right.$ for all $j \neq i$ ), the time derivative of their size

$$
\dot{x}_{i}=r_{i} x_{i}-c_{i} x_{i}^{2}
$$

is always negative. Finally, the third result says that rebels cannot go extinct if they are highly fanatic. Indeed, for small values of $x_{i}$ also the flows $V_{+i}$ and $V_{i+}$ are small, and again equation (13) can be approximated with equation (14). But the two flows of victims can be shown to be of the same order of $x_{i}$, so that sufficiently high values of $f_{i}$ guarantee that $\dot{x}_{i}$ is positive, and this prevents extinction.

Since the analytical treatment of the model is practically impossible, the only feasible approach is to study the model through numerical bifurcation analysis, or, more simply, through simulation. The results we have obtained so far with bifurcation analysis are still incomplete because the number of bifurcations is particularly high. Even in the simplest case $(N=2)$ the bifurcations of the model have not yet been fully understood. Thus, we present only results obtained through simulation (using a Dormand-Prince $(4,5)$ numerical scheme). In order to help the reader in drawing conclusions by comparing the various scenarios obtained through simulations, we present only results concerning conflicts among $N=3$ groups, more precisely one governmental group and two groups of rebels. This choice is due to the particular attention that this case has recently attracted worldwide.

Figure 3 is an example of the typical panel we can produce for any assignment of the parameters and of the initial conditions of the model. The figure summarizes the results of the simulation of a conflict among three groups. Group 1 is a governmental group $\left(r_{1}>0\right)$, while 2 and 3 are groups of rebels $\left(r_{i}<0, i=2,3\right)$. The initial conditions $x_{i}(0)$ are specified in the left part of the panel (here and in the following, $h_{i j}(0)=0$ ) together with the parameter values (the unit time is the day) characterizing military performances and recruitments. In the right part of the panel the time series of the group sizes $x_{i}$ are shown for a time period of 15 years. The time series clearly show that, in this example, the groups tend to a positive equilibrium. 


\begin{tabular}{|c|c|c|c|c|c|c|c|c|c|c|}
\hline$i$ & \multicolumn{3}{|c|}{$\overline{\sigma_{i j}^{s}}$} & \multicolumn{4}{|c|}{$\overline{\sigma_{i j}^{h}}$} & \multicolumn{3}{|c|}{$\overline{\tau_{i j}}$} \\
\hline 1 & $\bar{\square}$ & 050. & 05 & & & 0010 & .001 & $\square$ & 200 & 200 \\
\hline 2 & 0.1 & 0. & 005 & 0.02 & & 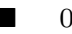 & .001 & 20 & $\mathbf{\square}$ & 20 \\
\hline 3 & 0.20 . & 05 & & 0.04 & 0 & 001 & & 20 & 20 & $\square$ \\
\hline$i$ & $r_{i}$ & $c_{i}$ & $\overline{\overline{d_{i}}}$ & $\overline{\overline{f_{i}}}$ & $\rho_{i}$ & $\overline{\bar{R}_{i}^{\max }}$ & & & & \\
\hline 1 & 0.025 & 0.025 & 0.5 & 0 & 1 & 0.25 & & & & \\
\hline 2 & -0.025 & 0 & 0 & 4 & 1 & 0.25 & & & & \\
\hline 3 & -0.05 & 0 & 0 & 5 & 1 & 0.25 & & & & \\
\hline
\end{tabular}

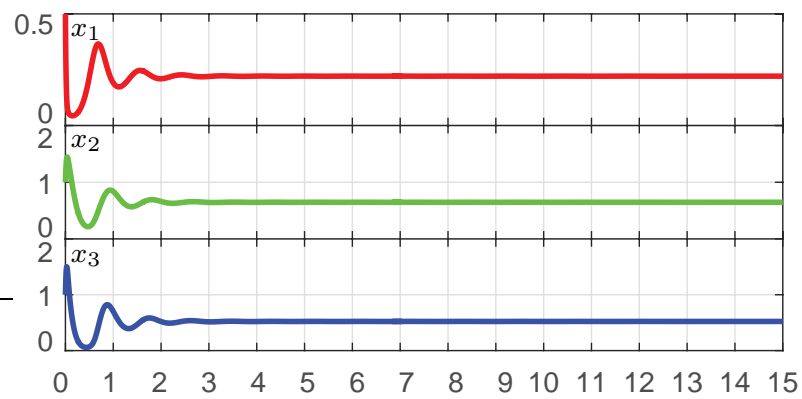

Fig. 3 Typical result of a simulation of model $(1-11,12 b, 13)$ where the three groups tend toward a positive equilibrium. Parameter values and initial conditions are specified on the left. They point out that 1 is a governmental group $\left(r_{1}>0\right)$, while 2 and 3 are groups of rebels $\left(r_{i}<0, i=2,3\right)$.

\section{Coexistence and eradication}

Depending upon initial conditions and parameter values the fate of the system can be different. If

$$
\lim _{t \rightarrow \infty} x_{i}(t)=0
$$

we say that the $i$-th group goes extinct or is eradicated.

One possible scenario is that no group is eradicated. This means that all groups remain in the game forever, or, in other words, that all groups coexist in the long term. However, the asymptotic dynamic regime can be different because coexistence can be stationary as in Figure 3, or periodic as in Figure 4. Notice that only the fanaticism of the rebels has been changed (actually increased) in passing from Figure 3 to Figure 4. Thus, the comparison of the two figures allows one to conclude that turbulent behaviors should be expected if rebels are highly fanatic.

\begin{tabular}{|c|c|c|c|c|c|c|c|c|c|c|}
\hline$i$ & \multicolumn{3}{|c|}{$\overline{\sigma_{i j}^{S}}$} & \multicolumn{4}{|c|}{$\overline{\sigma_{i j}^{h}}$} & \multicolumn{3}{|c|}{$\overline{\tau_{i j}}$} \\
\hline 1 & $\mathbf{\square} 0$. & 0050. & 005 & $\overline{\mathbf{D}}$ & & 001 & $\overline{0.001}$ & & 200 & 200 \\
\hline 2 & 0.1 & 0 . & 005 & $0.0^{\circ}$ & & $\square$ & 0.001 & 20 & $\square$ & 20 \\
\hline 3 & 0.20 . & 005 & 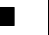 & 0.0 & & 001 & 口 & 20 & 20 & 口 \\
\hline$i$ & $\overline{r_{i}}$ & $\overline{\overline{c_{i}}}$ & $\overline{d_{i}}$ & $\overline{f_{i}}$ & $\rho_{i}$ & $\mathrm{R}_{i}^{\max }$ & $\overline{\overline{x_{i}}}$ & & & \\
\hline 1 & 0.025 & 0.025 & 0.5 & 0 & 1 & 0.25 & 0 & & & \\
\hline 2 & -0.025 & 0 & 0 & 8 & 1 & 0.25 & . & & & \\
\hline 3 & -0.05 & 0 & 0 & 9 & 1 & 0.25 & 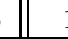 & & & \\
\hline
\end{tabular}

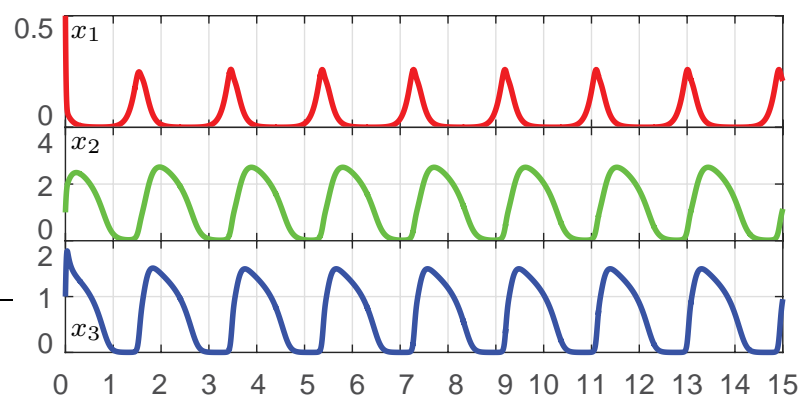

Fig. 4 Transients toward a periodic coexistence regime. Only the fanaticisms of the rebels are different then those of Figure 3. The comparison of the two figures shows that high fanaticisms of the rebels can trigger turbulent behaviours.

If one or more groups go extinct, the others coexist and tend to one of the possible asymptotic regimes. Figure 5 shows, for example, two cases in which one group goes extinct and two coexist. In the first panel the coexistence is stationary, while in the second it is periodic. The only difference between the two cases is that the efficiencies $\sigma_{21}^{h}$ of group 2 of rebels in capturing handling individuals of the governmental group 1 is higher in the second panel. Thus, the last five years of the time series of the second panel point out that a single group of rebels and a governmental group can tend toward a regime of periodic coexistence if the military efficiency of the rebels is sufficiently high. As far as we know, this is an original result in the theory of conflicts between two armed groups. 


\begin{tabular}{|c|ccc|ccc|ccc|}
\hline$i$ & \multicolumn{3}{|c|}{$\sigma_{i j}^{s}$} & \multicolumn{3}{|c|}{$\sigma_{i j}^{h}$} & \multicolumn{3}{|c|}{$\tau_{i j}$} \\
\hline 1 & $\mathbf{3}$ & 0.5 & 0.02 & $\mathbf{\square}$ & 0.2 & 0.01 & $\mathbf{\square}$ & 4 & 1 \\
2 & 0.25 & $\mathbf{0}$ & 0.07 & 0.1 & $\mathbf{\square}$ & 0.01 & 1 & $\mathbf{0}$ & 1 \\
3 & 0.01 & 0.02 & $\mathbf{\square}$ & 0 & 0 & $\mathbf{\square}$ & 2 & 1 & $\mathbf{\square}$ \\
\hline \hline$i$ & $r_{i}$ & $c_{i}$ & $d_{i}$ & $f_{i}$ & $\rho_{i}$ & $R_{i}^{\text {max }}$ & $x_{i}(0)$ \\
\hline 1 & 0.02 & 0.02 & 0.2 & 0 & 1 & 3 & 0.2 \\
2 & -0.1 & 0 & 0 & 6 & 1 & 3 & 0.2 \\
3 & -0.001 & 0 & 0 & 3 & 1 & 3 & 0.2 \\
\hline
\end{tabular}

\begin{tabular}{|c|ccc|ccc|ccc|}
\hline$i$ & \multicolumn{3}{|c|}{$\sigma_{i j}^{s}$} & \multicolumn{3}{|c|}{$\sigma_{i j}^{h}$} & \multicolumn{3}{|c|}{$\tau_{i j}$} \\
\hline 1 & $\mathbf{a}$ & 0.5 & 0.02 & $\mathbf{\square}$ & 0.2 & 0.01 & $\mathbf{\square}$ & 4 & 1 \\
2 & 0.25 & $\mathbf{\square}$ & 0.07 & 0.2 & $\mathbf{\square}$ & 0.01 & 1 & $\mathbf{\square}$ & 1 \\
3 & 0.01 & 0.02 & $\mathbf{\square}$ & 0 & 0 & $\mathbf{\square}$ & 2 & 1 & $\mathbf{\square}$ \\
\hline \hline$i$ & $r_{i}$ & $c_{i}$ & $d_{i}$ & $f_{i}$ & $\rho_{i}$ & $R_{i}^{\max }$ & $x_{i}(0)$ \\
\hline 1 & 0.02 & 0.02 & 0.2 & 0 & 1 & 3 & 0.2 \\
2 & -0.1 & 0 & 0 & 6 & 1 & 3 & 0.2 \\
3 & -0.001 & 0 & 0 & 3 & 1 & 3 & 0.2 \\
\hline
\end{tabular}
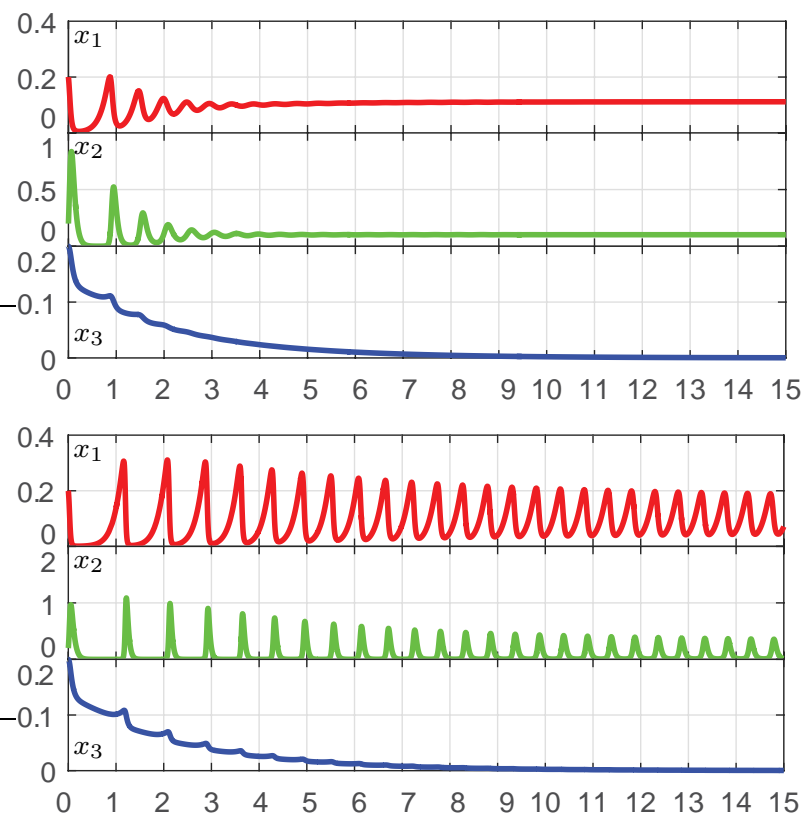

Fig. 5 Transients of three fighting groups (1 govermental, 2 and 3 rebels) with group 3 going extinct: top:stationary coexistence; bottom:periodic coexistence. Notice that $\sigma_{21}^{h}$ is the only parameter that is different in the two cases.

\section{Long term consequences of external shocks}

A short but heavy intervention of an external military force can vary quite significantly the number of individuals of one or more groups. Interventions of this kind, sometimes called shocks, can be interpreted as sudden perturbations of the state of the system. Typical interventions are the increase of the size of the group that the external military force wants to help, or the reduction of the size of its enemies. If the system has a unique attractor there are no consequences in the long term because the groups tend for all initial conditions to the same asymptotic regime. In other words, the system is resilient and the shock is smoothly absorbed. But if the system has multiple attractors, each one with its basin of attraction, the shock can have serious consequences even in the long term. This occurs when the shock entrains the jump of the state of the system from one basin of attraction to another.

Devising successful shocks is however quite difficult. Consider, for example, the case described in Figure 6 where, again, 1 is a governmental group and 2 and 3 are rebels. The time series show that in a relatively short time (3 years) the governmental group is almost annihilated while the rebels reach very high sizes. Imagine now that at the end of the fourth year an external country allied to the governmental group intervenes and reduces quite consistently the sizes of the two groups of rebels with a short but heavy military attack, as shown in Figure 6. The time evolutions of the groups after that intervention show that the shock is only partly successful. Indeed, the size of the governmental group rises up, but the rebels are not eradicated and the size of group 2 is actually slightly increased in the long term.

\begin{tabular}{|c|c|c|c|c|c|c|c|c|c|c|}
\hline$i$ & \multicolumn{3}{|c|}{$\overline{\sigma_{i j}^{s}}$} & \multicolumn{4}{|c|}{$\sigma_{i j}^{h}$} & \multicolumn{3}{|c|}{$\tau_{i j}$} \\
\hline 1 & $\square$ & 0.2 & 0.2 & & & 0.2 & 0.17 & & 66 & 58 \\
\hline 2 & 0.17 & 口 & 0.1 & & 15 & 口 & 0.12 & 18 & 口 & 86 \\
\hline 3 & 0.23 & 0.1 & 口 & & .2 & 0.03 & 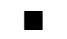 & 12 & 100 & $\mathbf{\square}$ \\
\hline$\overline{\bar{i}}$ & $r_{i}$ & $c_{i}$ & $\overline{d_{i}}$ & $\overline{f_{i}}$ & $\overline{\rho_{i}}$ & $\overline{R_{i}^{\max }}$ & & (0) & & \\
\hline 1 & 0.05 & 0 & 4 & 0 & 1 & $\frac{i}{0.25}$ & & 4 & & \\
\hline 2 & -0.03 & 0 & 2 & 4 & 1 & 0.25 & & 1 & & \\
\hline 3 & -0.01 & 0 & 2 & 3.2 & 1 & 0.25 & & 4 & & \\
\hline
\end{tabular}

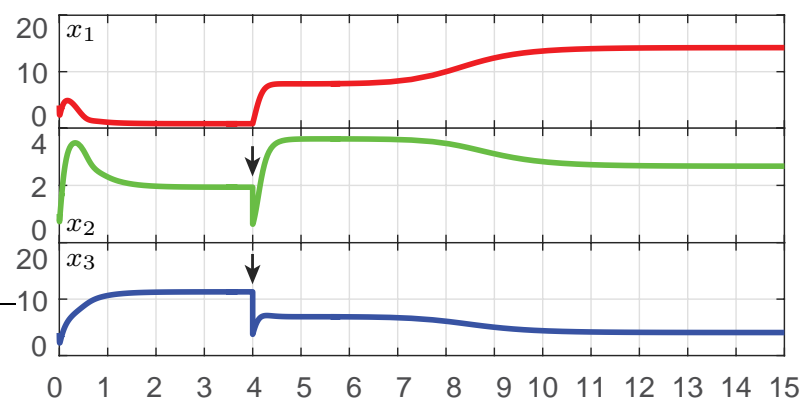

Fig. 6 Consequences of a contemporary external attack against groups 2 and 3, aimed at protecting the governmental group 1. 


\begin{tabular}{|c|ccc|ccc|ccc|}
\hline$i$ & \multicolumn{3}{|c|}{$\sigma_{i j}^{s}$} & \multicolumn{5}{|c|}{$\sigma_{i j}^{h}$} & \multicolumn{3}{|c|}{$\tau_{i j}$} \\
\hline 1 & $\mathbf{\square}$ & 1 & 0.08 & $\mathbf{0}$ & 0.4 & 0.02 & $\mathbf{\square}$ & 2 & 0.5 \\
2 & 0.5 & $\mathbf{\square}$ & 0.14 & 0.4 & $\mathbf{0}$ & 0.02 & 0.5 & $\mathbf{0}$ & 0.5 \\
3 & 0.04 & 0.04 & $\mathbf{0}$ & 0 & 0 & $\mathbf{\square}$ & 1 & 0.5 & $\mathbf{\square}$ \\
\hline \hline$i$ & $r_{i}$ & $c_{i}$ & $d_{i}$ & $f_{i}$ & $\rho_{i}$ & $R_{i}^{\text {max }}$ & $x_{i}(0)$ \\
\hline 1 & 0.04 & 0.04 & 0.2 & 0 & 1 & 6 & 0.2 & \\
2 & -0.2 & 0 & 0 & 6 & 1 & 6 & 0.5 & \\
3 & -0.02 & 0 & 0 & 3 & 1 & 6 & 0.3 \\
\hline
\end{tabular}

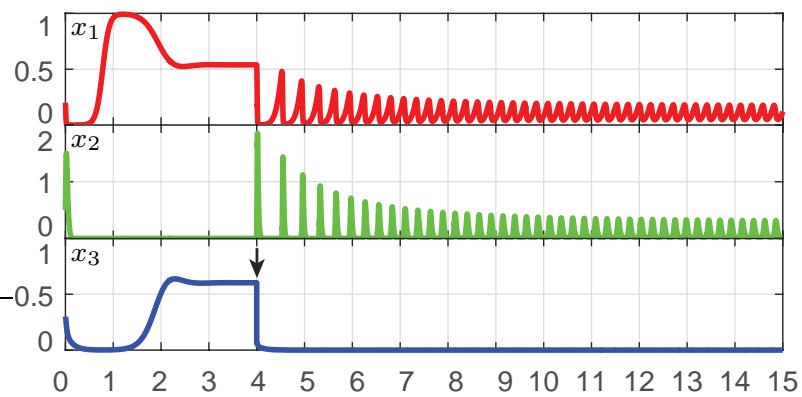

Fig. 7 Unpleasant consequences of an attack against a group of rebels (at the end of the 4th year): group 3 is attacked and eradicated, but the other group of rebels (group 2) reappears in the game (the new coexistence regime is periodic).

Figure 7 shows another surprising consequence of an external shock (given again after 4 years), with the aim of eradicating group 3, which is the only remaining dangerous group of rebels-group 2 being almost eradicated after 4 years. The result is in part as expected, namely group 3 is eradicated. But the surprise is that group 2 reappears in the game and reduces the average size of the governmental group.

It is worth noticing that the eradication of a group can, in principle, be obtained with a limited military effort, provided the system is shocked when its state is close to the boundary separating the two basins of attraction. For this reason, the success of a military intervention aimed at supporting one group might critically depend on the time of the intervention. Figure 8 shows an interesting example: the first scenario points out that without external intervention group 3 goes extinct, while the two bottom scenarios show the consequences of an intervention performed by an external country supporting group 3 of rebels by increasing their size $x_{3}$. On the left the intervention, performed after one year, is successful because group 3 does not go extinct anymore and group 2, in competition with group 3, is eradicated, while on the right the same intervention, performed after two years, is not successful because group 3 is still eradicated. The message of the numerical experiment described in Figure 8 is that the help to a group dangerously declining toward extinction has greater chances of success if the help is given earlier.

\begin{tabular}{|c|ccc|ccc|ccc|}
\hline$i$ & \multicolumn{3}{|c|}{$\sigma_{i j}^{s}$} & \multicolumn{3}{|c|}{$\sigma_{i j}^{h}$} & \multicolumn{3}{|c|}{$\tau_{i j}$} \\
\hline 1 & $\mathbf{\square}$ & 0.5 & 0.02 & $\mathbf{\square}$ & 0.2 & 0.01 & $\mathbf{\square}$ & 4 & 1 \\
2 & 0.25 & $\mathbf{\square}$ & 0.07 & 0.2 & $\mathbf{\square}$ & 0.01 & 1 & $\mathbf{\square}$ & 1 \\
3 & 0.01 & 0.02 & $\mathbf{\square}$ & 0 & 0 & $\mathbf{\square}$ & 2 & 1 & $\mathbf{\square}$ \\
\hline \hline$i$ & $r_{i}$ & $c_{i}$ & $d_{i}$ & $f_{i}$ & $\rho_{i}$ & $R_{i}^{\max }$ & $x_{i}(0)$ \\
\hline 1 & 0.02 & 0.02 & 0.2 & 0 & 1 & 3 & 0.2 \\
2 & -0.1 & 0 & 0 & 6 & 1 & 3 & 0.2 \\
3 & -0.001 & 0 & 0 & 3 & 1 & 3 & 0.2 \\
\hline
\end{tabular}
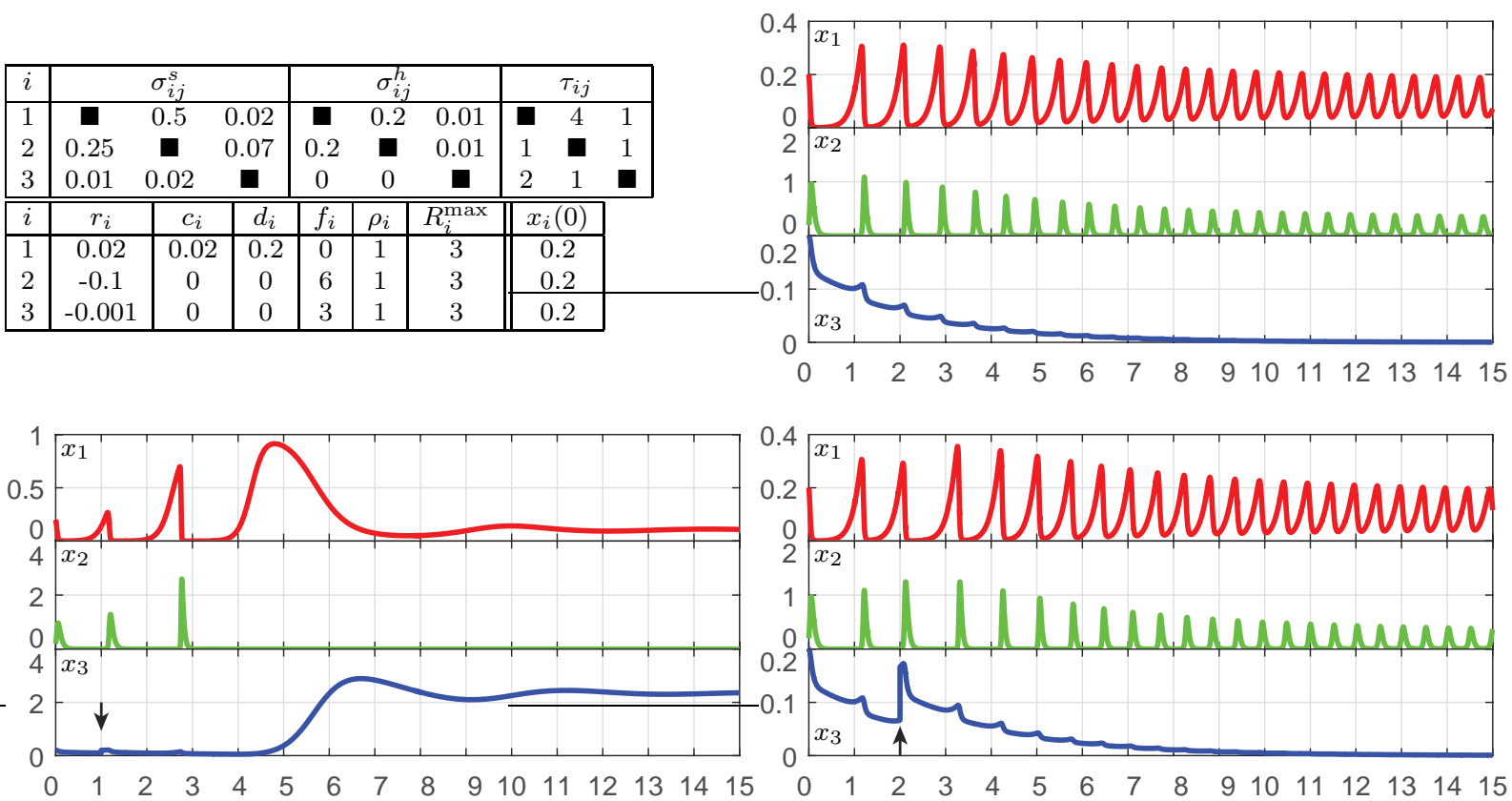

Fig. 8 Impacts of the same shock performed at two different times: bottom left: success (group 2 is eradicated and group 3 is sustained); bottom right: failure (group 2 is not eradicated and group 3 is not sustained). 


\section{Consequences of structural changes}

The military characteristics of a group can suddenly change and then remain constant for a long period, if not forever. The activation of a newly constructed military airport, the permanent supply of arms guaranteed by an external country, the establishment of a new intelligence system are examples of this kind of changes, from now on called structural.

The impact of structural changes can, in principle, be identified by simulating the model from the time $t^{*}$ of the change. The parameter values must be modified with respect to those used in the previous phase, i.e., for $t<t^{*}$, while the initial state must be equal to the state reached at $t=t^{*}$. Figure 9 shows, for example, the case in which the efficiencies $\sigma_{1 j}^{s}$ and $\sigma_{1 j}^{h}$ of the governmental group are doubled at $t=t^{*}=5$, when the groups are in a stationary coexistence regime. The impact of this structural change is absolutely not surprising: the new coexistence regime is still stationary but with a drop in the sizes of the rebels.

\begin{tabular}{|c|ccc|ccc|cc|c|}
\hline$i$ & \multicolumn{2}{|c|}{$\sigma_{i j}^{s} \cdot 10^{3}$} & \multicolumn{3}{|c|}{$\sigma_{i j}^{h} \cdot 10^{3}$} & \multicolumn{3}{|c|}{$\tau_{i j}$} \\
\hline 1 & $\mathbf{0}$ & 1 & 2 & $\mathbf{\square}$ & 0.25 & 0.5 & $\mathbf{\square}$ & 100 & 50 \\
2 & 4 & $\mathbf{a}$ & 0.2 & 1 & $\mathbf{0}$ & 0.05 & 50 & $\mathbf{\square}$ & 50 \\
3 & 8 & 0.2 & $\mathbf{0}$ & 2 & 0.05 & $\mathbf{a}$ & 50 & 50 & $\mathbf{\square}$ \\
\hline \hline$i$ & $r_{i}$ & $c_{i}$ & $d_{i}$ & $f_{i}$ & $\rho_{i}$ & $R_{i}^{\text {max }}$ & $x_{i}(0)$ \\
\hline 1 & 0.01 & 0.01 & 0.5 & 0 & 1 & 0.1 & 1 \\
2 & -0.01 & 0 & 0 & 4 & 1 & 0.1 & 0.5 \\
3 & -0.02 & 0 & 0 & 4.5 & 1 & 0.1 & 0.5 \\
\hline
\end{tabular}

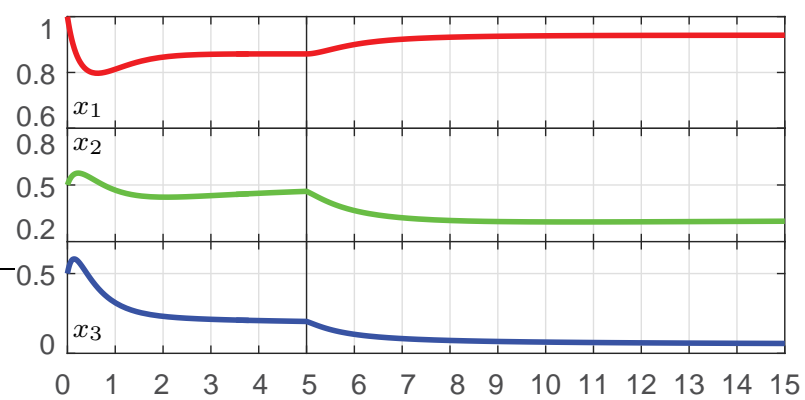

Fig. 9 The effect of doubling the efficiencies $\sigma_{1 j}^{s}$ and $\sigma_{1 j}^{h}$ of the governmental group 1 after 5 years.

Structural changes can also have relevant long term consequences. This actually occurs quite frequently because the system has so many bifurcations that it is easily possible that the perturbation of parameters interpreting the structural change implies the crossing of one or more bifurcation curves in parameter space. Figure 10 shows the consequences of three subsequent structural changes (corresponding to increases of the efficiencies $\sigma_{1 j}^{s}$ and $\sigma_{1 j}^{h}$ of group 1). The first change (occurring at the end of the $6^{\text {th }}$ year) stabilizes the system (switch from periodic to stationary coexistence), while the second (end of the $9^{\text {th }}$ year) eradicates group 3 and the third (end of the $11^{\text {th }}$ year) eradicates also group 2.

\begin{tabular}{|c|ccc|ccc|ccc|}
\hline$i$ & \multicolumn{4}{|c|}{$\sigma_{i j}^{s}$} & \multicolumn{4}{c|}{$\sigma_{i j}^{h}$} & \multicolumn{3}{|c|}{$\tau_{i j}$} \\
\hline 1 & $\mathbf{0}$ & 0.002 & 0.002 & $\mathbf{0}$ & 0.001 & 0.001 & $\mathbf{0}$ & 0.05 & 0.05 \\
2 & 20 & $\mathbf{\square}$ & 0 & 10 & $\mathbf{0}$ & 0 & 0.01 & $\mathbf{\square}$ & 0 \\
3 & 0 & 4 & $\mathbf{\square}$ & 0 & 2 & $\mathbf{0}$ & 0 & 0.05 & $\mathbf{\square}$ \\
\hline \hline$i$ & $r_{i}$ & $c_{i}$ & $d_{i}$ & $f_{i}$ & $\rho_{i}$ & $R_{i}^{\max }$ & $x_{i}(0)$ \\
\hline 1 & 2 & 2 & 1 & 0 & 1 & 2 & 1 & & \\
2 & -0.6 & 0 & 0 & 0.05 & 1 & 2 & 0.01 & & \\
3 & -0.02 & 0 & 0 & 2 & 1 & 2 & 0.05 \\
\hline
\end{tabular}

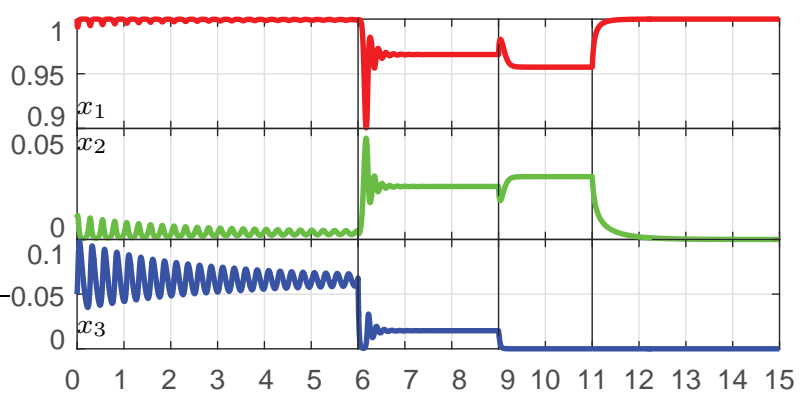

Fig. 10 The effects of three subsequent structural changes corresponding to increases of the efficiencies $\sigma_{1 j}^{s}$ and $\sigma_{1 j}^{h}$ of group 1. The first change (occurring at the end of the $6^{\text {th }}$ year) eliminates the oscillations (Hopf bifurcation), while the second and third (end of $9^{\text {th }}$ and $11^{\text {th }}$ years) eradicate groups of rebels (transcritical bifurcations).

\section{Conclusions}

The results presented in this paper allow us to conclude that the model we have proposed can produce extremely rich dynamic scenarios. In other words, our model can be interpreted as a general theory 
capable of explaining the great variety of phenomena historically observed in the context of armed conflicts. From a conceptual point of view, this is undoubtedly an important contribution.

The fate of real conflicts is often believed to be unpredictable because data are too scarce (see, for instance, [7] and [3] for studies on terrorism). But the truth is even worse than that. In fact, the results we have discussed show that the dynamics of fighting groups can be extremely sensitive to initial conditions and parameter values. Thus, the fate of a fight could actually be unpredictable even if very rich data sets support almost perfect estimates of the parameters. Indeed, a reason to tame the enthusiasm towards data-driven decision making.

We like to conclude this paper by stressing, once more, that the results we have presented are just preliminary. More effort is needed to answer important questions concerning conflicts among armed groups. Some of these questions are rather academic. For example: can the model have strange attractors, i.e., can the fate of conflicts be unpredictable in the rigorous mathematical sense? And, if yes, can peak-topeak dynamics exist and chaotic intermittence emerge? Other questions are of greater interest for decision makers. For example: can the model be used to predict, at least qualitatively, the effects of temporary coalitions, or to evaluate the impact of an increase of the power of the army or of the efficiency of the intelligence? Answering these questions requires a deep knowledge of the theory of nonlinear dynamical systems as well as a great competence in the numerical aspects of stability and bifurcation analysis, and is left for future research.

Acknowledgements The authors are grateful to Gustav Feichtinger and Jonathan Caulkins for their comments and encouragement. The help of three anonymous reviewers has allowed us to significantly improve the quality of the paper.

\section{References}

1. Kress, M.: Modeling armed conflicts. Science 336(6083), 865-869 (2012)

2. Lanchester, F.W.: Aircraft in warfare: The dawn of the fourth arm. Constable limited (1916)

3. Lopes, A.M., Machado, J.T., Mata, M.E.: Analysis of global terrorism dynamics by means of entropy and state space portrait. Nonlinear Dynamics 85(3), 1-14 (2016)

4. MacKay, N.J.: When Lanchester met Richardson, the outcome was stalemate: A parable for mathematical models of insurgency. Journal of the Operational Research Society 66(2), 191-201 (2015)

5. Richardson, L.F.: Mathematical psychology of war. W. Hunt (1919)

6. Richardson, L.F.: Mathematical psychology of war. Nature 135, 830-831 (1935)

7. Schiermeier, Q.: Attempts to predict terrorist attacks hit limits. Nature 517, 419-420 (2015) 